\title{
Temple and Postauricular Dissection in Face and Neck Lift Surgery
}

\author{
Joo Heon Lee ${ }^{1}$, Tae Suk Oh${ }^{2}$, Sung Wan Park 33 Jae Hoon Kim ${ }^{3}$, Tanvaa Tansatit ${ }^{4}$ \\ ${ }^{1}$ Area88 Plastic Surgery Clinic, Seoul; ${ }^{2}$ Department of Plastic Surgery, Asan Medical Center, University of Ulsan College of Medicine, Seoul; \\ ${ }^{3}$ April31 Plastic Surgery Clinic, Seoul, Korea; ${ }^{4}$ The Chula Soft Cadaver Surgical Training Center and Department of Anatomy, Faculty of \\ Medicine, Chulalongkorn University and King Chulalongkorn Memorial Hospital, Bangkok, Thailand
}

Periauricular paresthesia may afflict patients for a significant amount of time after facelift surgery. When performing face and neck lift surgery, temple and posterior auricular flap dissection is undertaken directly over the auriculotemporal, great auricular, and lesser occipital nerve territory, leading to potential damage to the nerve. The auriculotemporal nerve remains under the thin outer superficial fascia just below the subfollicular level in the prehelical area. To prevent damage to the auriculotemporal nerve and to protect the temporal hair follicle, the dissection plane should be kept just above the thin fascia covering the auriculotemporal nerve. Around the McKinney point, the adipose tissue covering the deep fascia is apt to be elevated from the deep fascia due to its denser fascial relationship with the skin, which leaves the great auricular nerve open to exposure. In order to prevent damage to the posterior branches of the great auricular nerve, the skin flap at the posterior auricular sulcus should be elevated above the auricularis posterior muscle. Fixating the superficial muscular aponeurotic system flap deeper and higher to the tympano-parotid fascia is recommended in order to avoid compromising the lobular branch of the great auricular nerve. The lesser occipital nerve ( $\mathrm{C} 2$, C3) travels superficially at a proximal and variable level that makes it vulnerable to compromise in the mastoid dissection. Leaving the adipose tissue at the level of the deep fascia puts the branches of the great auricular nerve and lesser occipital nerve at less risk, and has been confirmed not to compromise either tissue perfusion or hair follicles.

Keywords Rhytidoplasty / Paresthesia

Received: 17 Apr 2017 • Revised: 28 Jun $2017 \bullet$ Accepted: 29 Jun 2017

pISSN: 2234-6163 • elSSN: 2234-6171 • https://doi.org/10.5999/aps.2017.44.4.261 • Arch Plast Surg 2017;44:261-265
Correspondence: Joo Heon Lee Area88 Plastic Surgery Clinic, Yuri build 5th floor, 822 Seonreuong-ro, Gangnam-gu, Seoul 06014, Korea

Tel: $+82-2-548-0088$

Fax: +82-2-554-7557

E-mail: jj2197@naver.com

This article was presented at the 2017 Congress of the Korean Society of Aesthetic Plastic Surgery on April 2, 2017 in Seoul, Korea.

No potential conflict of interest relevant to this article was reported.

\section{INTRODUCTION}

Alopecia in the temple and posterior neck, hair line distortion,
ear deformity, and periauricular paresthesia can be minimized if
careful attention is paid to incision design, suturing both super-
ficial muscular aponeurotic system (SMAS) and skin flaps, and
precise undermining of subcutaneous flaps in facelift and neck- lift procedures. The spectrum of periauricular paresthesia is variable, including dysethesia and hypesthesia, which can afflict patients for a significant amount of time after facelift surgery. Sensation in the ear and periauricular region is governed by the great auricular nerve, lesser occipital nerve, auriculotemporal nerve, and vagus nerve (Fig. 1) [1]. The auriculotemporal nerve provides sensory innervation to the temple area. It has long 

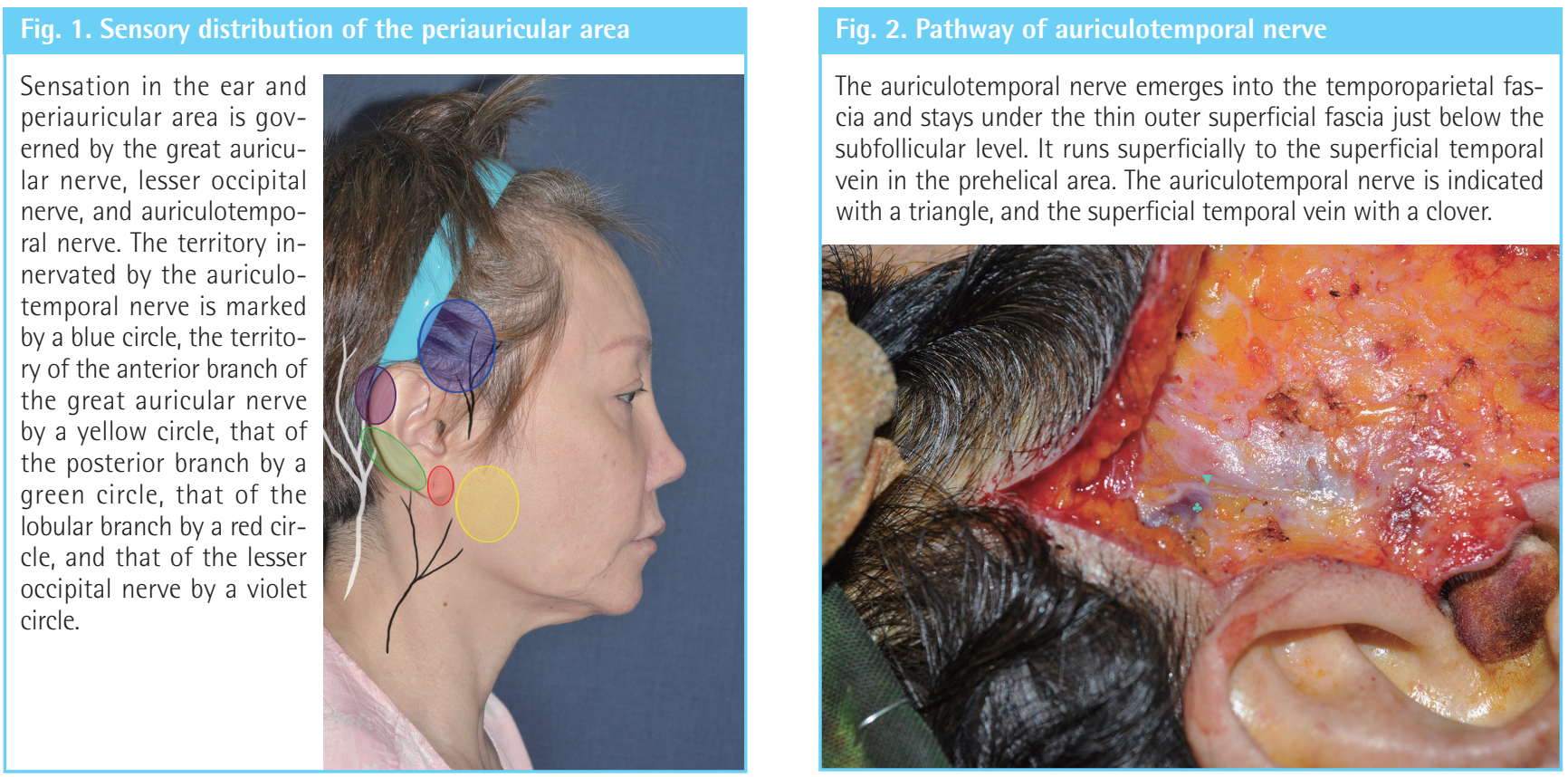

\section{Fig. 3. Detailed plane of auriculotemporal nerve}

The auriculotemporal nerve travels just beneath the thin outer superficial fascia in the subfollicular level. It is easier to protect the auriculotemporal nerve in the preauricular dissection when dissecting above the thin fascia covering the nerve to initiate the prehelical dissection from the upper pole of the helix. The auriculotemporal nerve is indicated with a triangle and the subfollicular thin fascia with a heart. (A) intra operative view. (B) schematic view.
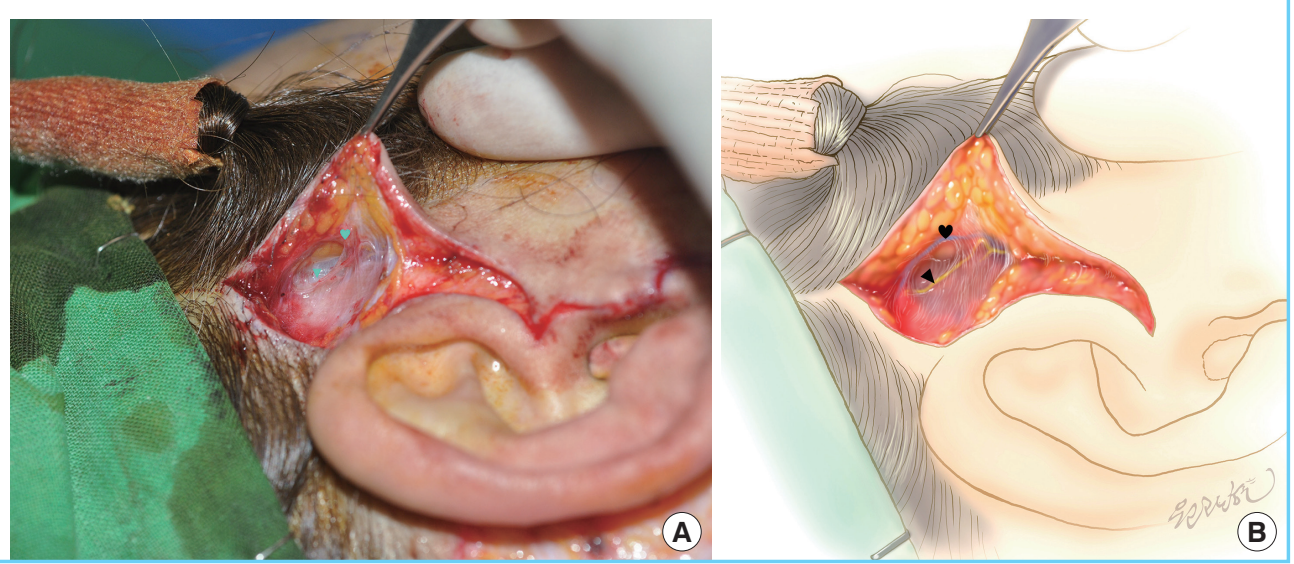

been stated that the great auricular nerve governs sensation in the lower two-thirds of the helix [1-3], whereas the upper onethird is governed by the lesser occipital nerve. However, the lesser occipital nerve has been revealed to innervate the helix more widely than expected [4], and the ear lobe has been found to receive sensory innervation independently via the lobular branch of the great auricular nerve [5]. In order to prevent periauricular paresthesia during facelift, the surgeon should elevate the postauricular flap precisely, with a thorough understanding of the pathways of these sensory nerves and of fascial relationships in the periauricular region. During periauricular dissection in facelift surgery, in addition to minimizing nerve trauma, vascular perfusion to the skin flap should be ensured, and the hair follicles of the temple and mastoid are to be protected.

\section{TEMPLE DISSECTION}

The auriculotemporal nerve, which arises from the maxillary nerve (V2), innervates the temporal skin. The nerve runs parallel $1-1.5 \mathrm{~cm}$ anterior to the helix across the temporal vein and runs the underneath the temporal scalp. The temporal vein travels in the midst of the superficial temporal fascia, and the auriculotemporal nerve seems to run superficial in the superficial temporal fascia. The nerve stays under the thin outer superficial fascia just below the subfollicular level in the distal part of the temporal scalp (Fig. 2). To prevent damage to the auriculotemporal nerve and to protect temporal hair follicles, the surgeon should keep the dissection plane just above the thin fascia covering the auriculotemporal nerve and temporal vein. It is easier to protect the auriculotemporal nerve in the preauricular dissection when 


\section{Fig. 4. Exposure of the auriculotemporal nerve}

In cases of secondary facelift or when threads have previously been inserted, the thin fascia under the hair follicle is likely to be disrupted. The auriculotemporal nerve is indicated with a triangle.

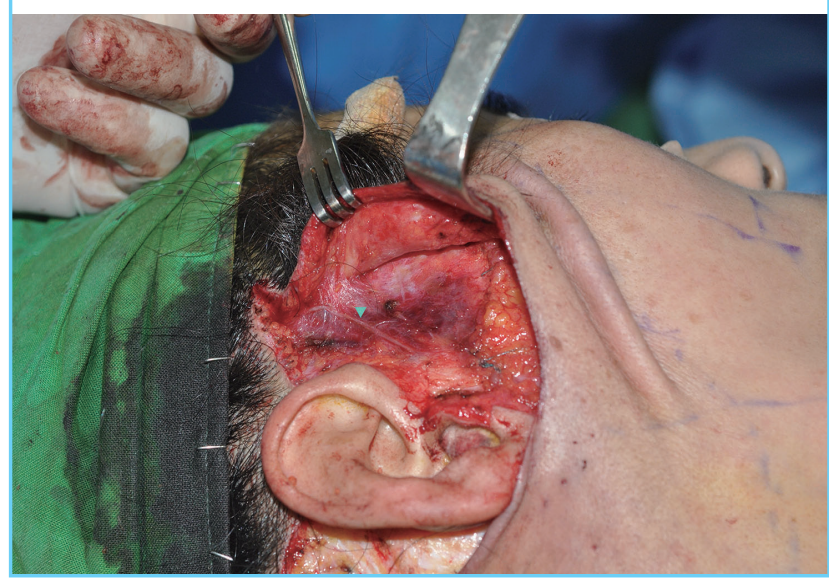

dissecting above the thin fascia covering the nerve to initiate the prehelical dissection from the upper pole of the helix (Fig. 3). In cases where threads have previously been inserted and in secondary facelifts, the thin outer superficial fascia under the hair follicle is very likely to be disrupted, resulting in exposure of the auriculotemporal nerve (Fig. 4). More careful temple dissection is needed to minimize the damage to the auriculotemporal nerve in these cases.

\section{RETROAURICULARIDISSECTION}

\section{Pathway of the great auricular nerve and the lesser occipital nerve}

The great auricular nerve (C2, C3) and the lesser occipital nerve (C3) govern periauricular sensation. The great auricular nerve is a sensory nerve that arise from $\mathrm{C} 2$ and $\mathrm{C} 3$. It runs behind the sternocleidomastoid (SCM) muscle approximately 1 $\mathrm{cm}$ below the spinal accessory nerve. It emerges into the midbody of the SCM muscle, staying in the muscle fascia (Fig. 5), and then crosses the SCM muscle, where it resides in its most superficial location (in the superficial investing fascia of the neck) before arborizing into 2 or 3 branches. As McKinney postulated, the nerve is most vulnerable to be compromised during retroauricular dissection in this area (the McKinney point) (Fig. 5) [3]. The anterior branch continues in a plane between the SCM muscle and the parotid gland prior to its terminal, which provides sensation to the skin overlying the parotid gland and anteroinferior aspect of the auricle [6]. The posterior branch travels under the SMAS, namely the auricularis posterior muscle, before reaching the posterior auricle (Fig. 5) [7]. The lobular branch most frequently originates from a common trifurca-

\section{Fig. 5. Pathway of the great auricular nerve}

The great auricular nerve emerges into the mid-body of the sternocleidomastoid (SCM) muscle, staying in the muscle fascia, and then crosses the SCM muscle, where it resides just under the deep fascia before arborizing into branches. The posterior branch travels under the superficial musculoaponeurotic system, in particular the auricularis posterior muscle, before reaching the posterior auricle. The muscle fascia of the SCM muscle is indicated by a spade, the deep fascia by a triangle, the posterior branch by star, and the posterior auricularis muscle by an arrow.

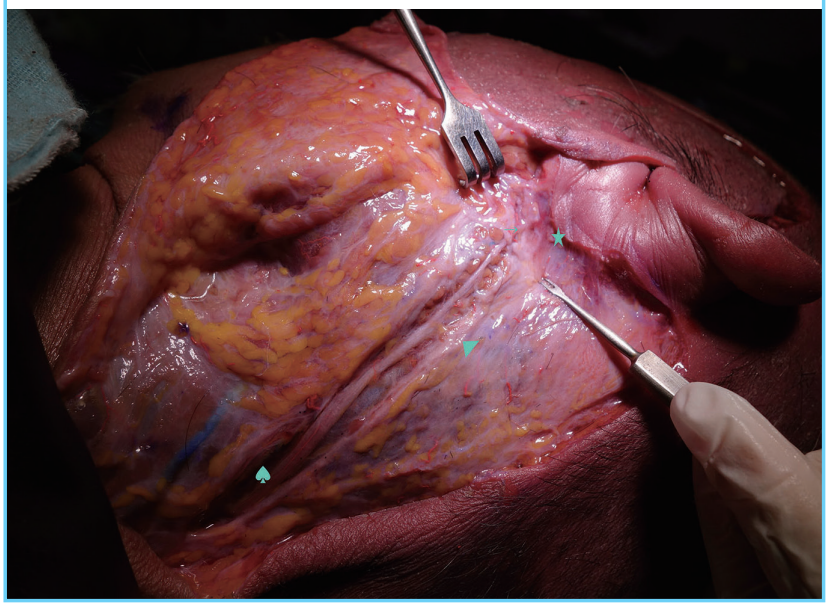

tion with the anterior and posterior branches. It gives sensation to the lobule, located in the center of the antitragus and directly inferior to the caudal edge of the tragus [8]. Unlike the pathway of the anterior branch, the lobular branch seems not to go through the parotid gland, but rather travels through the superficial plane to reach the antitragus [5]. The lesser occipital nerve is a purely sensory nerve from the ascending superficial branch of the second and, occasionally, the third ventral ramus of the cervical plexus. It curves lateral to the accessory nerve and ascends along the posterior border of the SCM muscle, sending anterior branches to the ear and posterior branches to the mastoid area. The anterior branches overlap with the posterior branches of the great auricular nerve, and the posterior branches of the lesser occipital nerve overlap with the great occipital nerve [1]. The lesser occipital nerve, after piercing the deep cervical fascia, consistently emerges behind the posterior border of the SCM muscle superior to the great auricular nerve, then courses in an oblique-cephalic direction toward the ear and the mastoid, diverging in a fan-like fashion from the great auricular nerve. The nerve runs between the muscle fascia and the SMAS. It enters the subcutaneous plane at a proximal and variable level [4], although in some patients it travels superficially throughout the entirety of its course, and thus stays in a position vulnerable to compromise during mastoid dissection. 


\section{POSTAURICULARDISSECTION}

When performing rejuvenation surgery of the face and neck, posterior auricular flap dissection is performed directly over the lesser occipital and great auricular nerve territory, with potential damage to the nerves. Wide posterior auricular subcutaneous dissection is essential to obtain an aesthetically balanced result in various kinds of rhytidectomy. The great auricular nerve has been reported to be the most commonly injured sensory nerve after facelift. The McKinney point is well known as the most common site where the great auricular nerve is injured, as the great auricular nerve travels in a more superficial plane that is prone to exposure during subcutaneous flap dissection. The deep fascia of the posterior auricular area is covered by well-defined adipose tissue [7], although it may be thin in slender patients. The adipose tissue around the McKinney point has a denser fascial relationship with the skin than with the underlying deep fascia. The adipose tissue covering the deep fascia is apt to be elevated inadvertently from the deep fascia due to the denser relationship of the fascia with the skin, which leaves the great auricular nerve vulnerable to exposure during posterior auricular flap dissection (Fig. 6). When performing antegrade dissection of a posterior auricular flap, the surgeon should be cautious to leave the adipose tissue on the deep fascia around the McKinney point. The anterior branch of the great auricular nerve is well protected as it travels deep into the parotid gland. The posterior branches of the great auricular nerve travel beneath the SMAS, and in particular, the auricularis posterior muscle is near the innervation area [7]. To prevent damage to the posterior branches, the skin flap at posterior auricular sulcus should be elevated above the auricularis posterior muscle (Fig. 7). The lobular branch of the great auricular nerve is apt to be

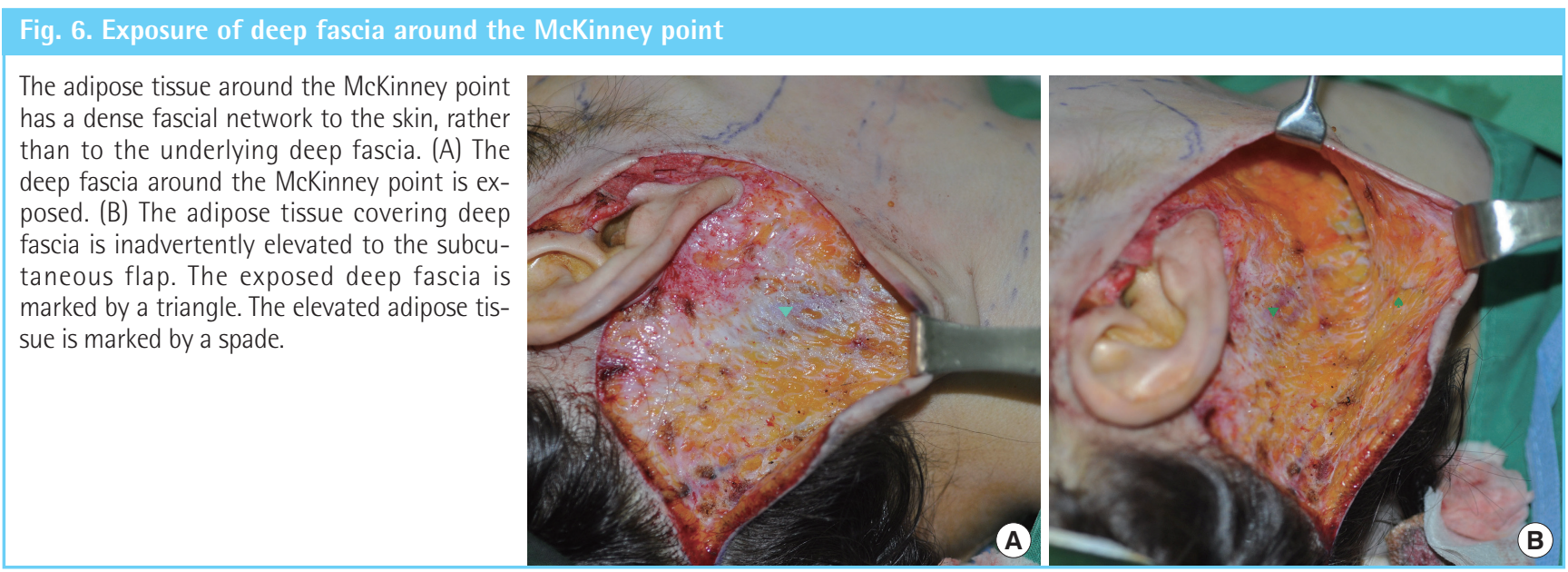

Fig. 7. Recommended postauricular subcutaneous flap elevation 1

For preventing damage to the posterior branches, the skin flap at posterior auricular sulcus should be elevated above the auricularis posterior muscle. The auricularis posterior muscle is marked by a star.

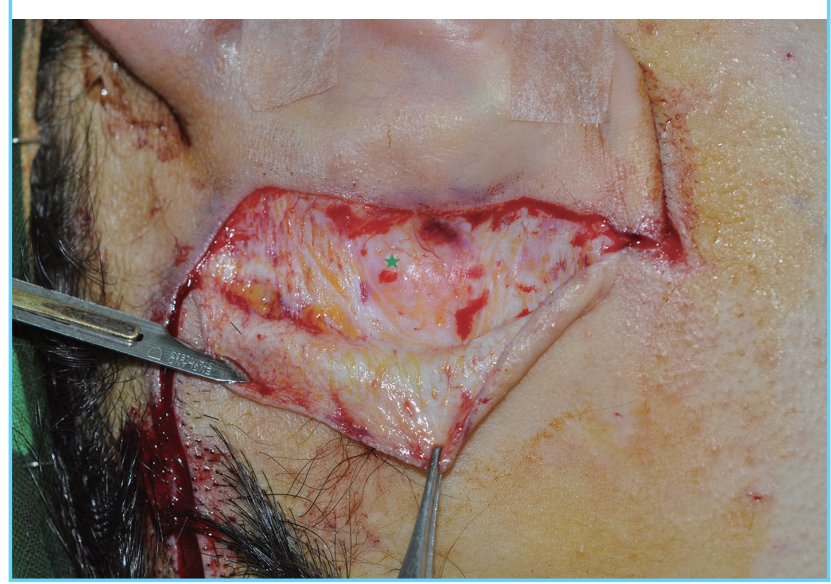

Fig. 8. Recommended postauricular subcutaneous flap elevation 2

Leaving the adipose tissue down to the deep fascia puts the branches of great auricular nerve and lesser occipital nerve at less risk, and avoids compromising tissue perfusion.

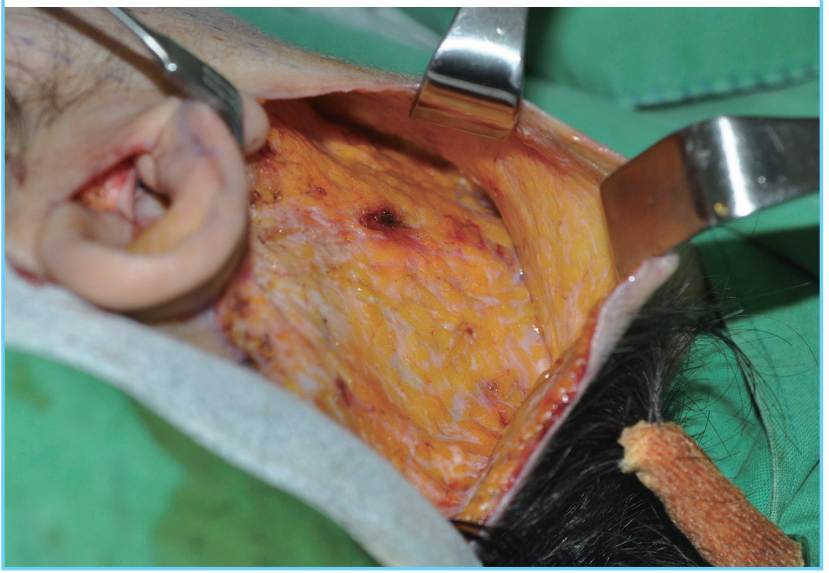


compromised when an SMAS flap is fixated to the tympano-parotid fascia. The lobular branches travel superficially to the depth of the tympano-parotid fascia and enter the lobule through the lower half of the tympano-parotid fascia; therefore, it is strongly recommended that the SMAS flap should be fixated deeper and higher to the tympano-parotid fascia. The plane of the great auricular nerve pathway becomes the subcutaneous level at the more distal portion of the terminal branches; however, the lesser occipital nerve enters the subcutaneous plane at a proximal and variable level [4]. To preserve robust blood supply to the posterior auricular flap, many surgeons straightforwardly perform the dissection at the fascial level. In our experience, dissecting at the fascial level in the postauricular area does not always guarantee protecting the lesser auricular nerve. Leaving the adipose tissue at the level of the deep fascia puts the branches of the great auricular nerve and lesser occipital nerve at less risk, and has been confirmed not to compromise either tissue perfusion or hair follicles (Fig. 8).

\section{CONCLUSIONS}

Alopecia and paresthesia in the periauricular area often afflict patients who have undergone facelift and necklift procedures. A thorough understanding of the periauricular anatomy, including the pathway, course, and plane of the auriculotemporal nerve, great auricular nerve, and lesser occipital nerve is essential to avoid compromising the nerves and hair follicles, as well as to guarantee robust flap perfusion. Additionally, awareness of the adipose tissue covering the mastoid fascia and its fascial relationships might lead the surgeon to more successful outcomes in facelift and necklift surgery.

\section{REFERENCES}

1. Berry M, Bannister HL, Standring MS. Nervous system. In: Gray H, Williams PL, Bannister LH, editors. Gray's anatomy. 38 ed. Edinburgh: Churchill Livingstone; 1995. p.123864.

2. McKinney P, Katrana DJ. Prevention of injury to the great auricular nerve during rhytidectomy. Plast Reconstr Surg 1980;66:675-9.

3. McKinney P, Gottlieb J. The relationship of the great auricular nerve to the superficial musculoaponeurotic system. Ann Plast Surg 1985; 14:310-4.

4. Pantaloni M, Sullivan P. Relevance of the lesser occipital nerve in facial rejuvenation surgery. Plast Reconstr Surg 2000;105:2594-9.

5. Sharma VS, Stephens RE, Wright BW, et al. What is the lobular branch of the great auricular nerve? Anatomical description and significance in rhytidectomy. Plast Reconstr Surg 2017;139:371e-378e.

6. Lefkowitz T, Hazani R, Chowdhry S, et al. Anatomical landmarks to avoid injury to the great auricular nerve during rhytidectomy. Aesthet Surg J 2013;33:19-23.

7. Rohrich RJ, Taylor NS, Ahmad J, et al. Great auricular nerve injury, the "subauricular band" phenomenon, and the periauricular adipose compartments. Plast Reconstr Surg 2011; 127:835-43.

8. Hu J, Ye W, Zheng J, et al. The feasibility and significance of preservation of the lobular branch of the great auricular nerve in parotidectomy. Int J Oral Maxillofac Surg 2010;39: 684-9. 